\title{
La deuxième édition des "Rhapsodies" de Pétrus Borel
}

\section{Michel Brix}

\section{(2) OpenEdition}

1 Journals

Édition électronique

URL : http://journals.openedition.org/studifrancesi/10119

DOI : 10.4000/studifrancesi.10119

ISSN : 2421-5856

Éditeur

Rosenberg \& Sellier

\section{Édition imprimée}

Date de publication : 1 décembre 2017

Pagination : 487-489

ISSN : 0039-2944

\section{Référence électronique}

Michel Brix, "La deuxième édition des "Rhapsodies" de Pétrus Borel », Studi Francesi [En ligne], 183 (LXI | III) | 2017, mis en ligne le 01 décembre 2018, consulté le 22 janvier 2021. URL : http:// journals.openedition.org/studifrancesi/10119; DOI : https://doi.org/10.4000/studifrancesi.10119

\section{(c) (†) $\odot$}

Studi Francesi è distribuita con Licenza Creative Commons Attribuzione - Non commerciale - Non opere derivate 4.0 Internazionale. 


\author{
La deuxième édition \\ des "Rhapsodies" de Pétrus Borel
}

\begin{abstract}
The scholars don't agree when they list the bibliographical advertisements which appear on the second edition (1833) of Rhapsodies, the poetical collection that French writer Pétrus Borel published at the time of the "Petit Cénacle". The detailed examination of the only copy known of this second edition shows that the publisher of the book reused the covers of the Rhapsodies' first edition and turned them over. In that way, we both find, in the second edition, the bibliographical advertisements which appeared in the first edition (and which were thus obsolete in 1833 ) and a few bibliographical advertisements which really concern the year 1833 . The article clarifies which are the former, and which are the latter.
\end{abstract}

La «bibliographie matérielle» s'inspire de la «physical bibliography», que les Anglais ont développée dans le cadre de leurs études sur les premières éditions des œuvres de Shakespeare, et qui correspond plus ou moins à la «codicologie» pratiquée par les médiévistes: il s'agit de s'intéresser à tous les paramètres matériels de la production d'un livre, comme la nature, l'origine, la trame et le filigrane du papier, les gravures, ou encore la typographie (ou l'écriture, dans le cas des manuscrits du Moyen Âge). En France, on a souvent recours à ces méthodes pour examiner les ouvrages parus aux $\mathrm{XVI}^{e}$, XVII et $\mathrm{XVIII}{ }^{e}$ siècles: les spécialistes se trouvent ainsi en mesure de détecter les contrefaçons, lorsque celles-ci ne s'affichent pas comme telles mais sont "déguisées" en éditions originales; ils peuvent également vérifier si les indications figurant sur la page de titre - date, lieu d'édition, nom de l'éditeur - sont bien exactes (les indications fausses étaient nombreuses pendant les périodes où la censure s'exerçait de façon sévère); enfin, les études de bibliographie matérielle - portant sur la comparaison du plus grand nombre possible d'exemplaires d'un ouvrage - ont permis d'établir que ces exemplaires, même issus d'un et un seul tirage, n'étaient pas nécessairement tous identiques entre eux, puisque des "cartons" (c'est-à-dire des feuillets corrigés) pouvaient remplacer des pages fautives dans les derniers exemplaires traités par le brocheur ou le relieur.

Mais pareils examens «matériels» ne sont pas moins nécessaires en ce qui concerne la production littéraire du XIX siècle, comme l'atteste le cas de la deuxième édition des Rhapsodies, recueil poétique de Pétrus Borel. La première édition de ce recueil parut chez le libraire parisien Levavasseur (installé au Palais-Royal), porte la date de 1832 et se trouve enregistrée dans la Bibliographie de la France du 31 décembre 1831. Ce recueil est célèbre, notamment, parce qu'il cite dans sa préface tous les jeunes artistes ou écrivains qui participaient, avec l'auteur, aux réunions du "Petit Cénacle" (Jean Duseigneur, Napoléon Thomas, Gérard [de Nerval], Joseph Bouchardy, Théophile Gautier, Alphonse Brot, Auguste Maquet [Augustus Mac-Keat], Jules Vabre, Léon Clopet, Théophile Dondey [O'Neddy] ainsi qu'un certain Vigneron). Autres informations précieuses que l'on retire de la consultation de ce volume: en quatrième de couverture, figure une liste d'ouvrages «sous presse» et «à paraître incessamment», qui fait la part belle aux compagnons cités dans la préface. Voici ladite liste (on notera que la majorité de ces titres sont fantaisistes): 
PÂTURE À LISEURS, par Pétrus Borel, 1 vol. in- $8^{\circ}$, orné de vignettes de Napol[éon] Thom[as] et de Joseph Bouchardy. 7 f. 50 c.

Du même auteur, APPEL AUXJEUNES FRANÇAIS À CCEUR DE LION, brochure in- $8^{\circ} .2 \mathrm{f}$. ODELETTES et ÉTUDES DRAMATIQUES, par Gérard.

MOSAÏQUES, par Philadelphe O’Neddy.

ODES ARTISTIQUES, par Théophile Gautier.

MATER DOLOROSA, par Augustus Mac-Keat.

ESSAI SUR L'INCOMMODITÉ DES COMMODES, par Jules Vabre.

Le volume borélien des Rhapsodies eut les honneurs d'une deuxième édition, qui est beaucoup moins bien connue que la première. Cette deuxième édition a paru chez Bousquet (qui reprit une partie du fonds Levavasseur) et porte la date de 1833, mais on ignore à quelle période elle a plus précisément été mise en vente (la publication dut être au moins discrète, puisqu'elle n'a pas fait l'objet d'un enregistrement dans la Bibliographie de la France). Le frontispice de Joseph Bouchardy ornant la première édition (Bouchardy fut graveur avant de devenir un auteur dramatique) est remplacé dans la deuxième par un encadrement du titre gravé à l'eau-forte (où on voit la mention: « $2^{\mathrm{e}}$ édition») et réalisé par Célestin Nanteuil. Sur la couverture figure aussi la mention «Deuxième édition» et - sous le nom de l'auteur - apparaît la formule «Champavert le lycanthrope». À l'intérieur de l'ouvrage, le texte est identique, mais on a ajouté, en tête de cette deuxième édition, un cahier initial (numéroté $\mathrm{V}-\mathrm{X})^{1}$ reproduisant partiellement la «Notice sur Champavert» qui ouvre le recueil Champavert, contes immoraux, paru en février ou mars $1833^{2}$.

Les affirmations des critiques se mettent cependant à diverger dès que l'on évoque les annonces figurant sur la couverture de l'édition Bousquet des Rhapsodies. Je me limiterai à trois exemples. En 1881, A. Parran, auteur d'un répertoire bibliographique consacré à Borel et à Dumas, affirme que se trouve annoncé sur la couverture de la deuxième édition des Rhapsodies: «Du même auteur: - Faust, dauphin de France; un fort volume in $-8^{\circ} \gg^{3}$. En 2002, dans la biographie qu'il consacre au lycanthrope $e^{4}$, Jean-Luc Steinmetz dresse la liste des publications qui figurent en «quatrième de couverture» de l'édition Bousquet des Rhapsodies: or - sans que J.-L. Steinmetz paraisse le remarquer -, il s'avère que ces annonces s'identifient mot pour mot à celles que l'on voit au dos de l'édition Levavasseur. Enfin, on relève cette dernière mention, dans la récente biographie de Théophile Gautier par Stéphane Guégan: «Début 1833, les Contes du bousingo [sont] annoncés sur la quatrième de couverture de la seconde édition des Rhapsodies de Borel» ${ }^{5}$.

Pourquoi ces affirmations ne concordent-elles pas entre elles? Comment le volume peut-il porter à la fois les annonces de la première édition (qui remplissaient la totalité

(1) Ce cahier initial contient aussi la nouvelle page de titre.

(2) Paris, Renduel, 1833 (enregistrement dans la Bibliographie de la France du 2 mars 1833). Cette «Notice sur Champavert» (ou «Notice sur Pétrus Borel. - Champavert le Lycanthrope $\rightarrow$, selon le titre qui apparaît dans la deuxième édition des Rhapsodies) explique que «Pétrus Borel» est le pseudonyme d'un écrivain qui s'appelle en fait Champavert, et dont le suicide est raconté dans le dernier conte qui forme le recueil Champavert.

(3) A. PARRAn, Romantiques. Éditions originales, vignettes, documents inédits ou peu connus, Alais, Imprimerie J. Martin, 1881, p. 6.

(4) Pétrus Borel. Vocation: "poète maudit" (Paris, Fayard, 2002), p. 110.

(5) St. GuÉgan, Théophile Gautier, Paris, Gallimard, 2011, p. 66 (on sait que les Contes du bousingo, qui ne parurent jamais, furent plusieurs fois annoncés et devaient réunir des récits venant de tous les membres "écrivains" du Petit Cénacle). 
de la quatrième de couverture), et plusieurs annonces supplémentaires? La réponse à ces questions réside dans les détails de la fabrication même de la deuxième édition des Rhapsodies. Si l'on s'en tient aux collections publiques, il semble ne subsister aujourd'hui qu'un seul exemplaire de cet ouvrage, en l'occurrence celui qui appartient au fonds Lovenjoul de la Bibliothèque de l'Institut, à Paris 6 . Quand on ouvre ledit exemplaire unique, on voit immédiatement que l'éditeur Bousquet a procédé de manière très inhabituelle pour composer les couvertures. À l'évidence, Bousquet avait encore dans sa boutique un stock de couvertures non utilisées (et donc non encore pliées) de l'édition précédente. Ainsi - pour réduire les frais -, il a manifestement ordonné de récupérer et de recycler ces couvertures en les retournant et en imprimant les nouvelles mentions au dos, avant de les plier en faisant en sorte que l'on voie ces mentions nouvelles, et non les anciennes, quand le livre est fermé. C'est ainsi qu'au verso de la première de couverture, on trouve l'ancienne quatrième de couverture, avec les annonces (qui ne se trouvent donc dans cette édition que par la grâce du "recyclage" des anciennes couvertures). De même, en feuilletant jusqu' au bout l'exemplaire de l'Institut, on a la surprise de voir, sur la troisième de couverture (c'est-à-dire au recto de la quatrième), ... la première de couverture de l'édition précédente, à la date de 1832 et à l'enseigne de Levavasseur! Enfin, la quatrième de couverture de l'édition Bousquet contient effectivement des annonces, inédites cette fois, qui concernent la librairie Renduel et la librairie Paulin et qui ont bien été rédigées au moment de la confection du volume ${ }^{7}$. Comme ces annonces n'ont jamais, à ma connaissance, été reproduites in extenso (ainsi Parran et Guégan ne les citent que très partiellement), je prends l'initiative de les transcrire toutes ci-dessous:

Librairie d'Eugène Renduel, rue des Grands-Augustins, n. 22.

En vente du même auteur:

CHAMPAVERT LE LYCANTHROPE, Contes immoraux, un vol. in- $8^{\circ}$, orné de vignettes de Gigoux.

Sous presse:

FAUST, DAUPHIN DE FRANCE, un fort volume in- $8^{\circ}$.

Pour paraître le 15 janvier:

CONTES DU BOUSINGO, par une camaraderie. - In- $8^{\circ}$, ornés de vignettes par Célestin Nanteuil. Les deux premiers volumes sont sous presse.

Librairie de Paulin, place de la Bourse.

ALBERTUS, OU L'ÂME ET LE PÉCHÉ, légende théologique, par Théophile Gautier.

On note, entre autres, que - contrairement à l'affirmation d'A. Parran - Faust, dauphin de France n'a jamais été annoncé comme une œuvre de Pétrus Borel.

MICHEL BRIX

Université de Namur

(6) Où il porte la cote LOV 13802.

(7) Il n'était pas exceptionnel, à l'époque, que les annonces figurant en quatrième de couverture d'un livre concernassent un autre éditeur. On trouve également des annonces «Renduel» au dos de l'édition d'Albertus, ou l'Âme et le péché, légende théologique de Gautier (1833), alors que cet ouvrage était publié chez Paulin, place de la Bourse. 